\title{
Consumo e dispersão secundária de sementes de Mucuna urens (Fabaceae) em Floresta Atlântica no Sul do Brasil
}

Consumption and secondary dispersal of seeds of Mucuna urens (Fabaceae) in the Atlantic Forest in Southern Brazil

\author{
Thalita Gabriella Zimmermann, ${ }^{1,4}$ Romualdo Morelatto Begnini, ${ }^{2}$ Tânia Tarabini Castellani, ${ }^{2}$ \\ Benedito Cortês Lopes ${ }^{2} \&$ Ademir Reis ${ }^{3}$
}

\begin{abstract}
Resumo
No presente estudo foram avaliados o consumo, a remoção e a dispersão secundária de sementes de Mucuna urens (Fabaceae - Faboideae) em um fragmento de Floresta Atlântica, no Parque Municipal da Lagoa do Peri, Florianópolis, SC. O padrão biométrico e o consumo por invertebrados foram avaliados em 100 sementes. A remoção e a dispersão secundária foram avaliadas em 120 e 25 sementes, respectivamente. A média do

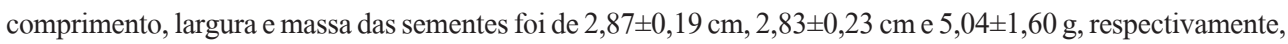
e $41 \%$ estavam consumidas por besouros Curculionidae, Scolytinae. Não houve relação do comprimento e da largura das sementes consumidas e não consumidas, sendo a massa das consumidas significativamente menor do que as não consumidas, devido aos escolitíneos se alimentarem do cotilédone. As cutias (Dasyprocta azarae) removeram $74,16 \%(\mathrm{n}=89)$ das sementes. Esse roedor dispersou $48 \%$ e enterrou $36 \%$ das sementes, a uma distância média de 8,06 m ( \pm 7,46 m), variando de 1,00 a 35,90 m. A dispersão secundária e o estocamento das sementes de $M$. urens por cutias diminui a probabilidade de predação por outros vertebrados e invertebrados e aumenta as chances de ocorrer a germinação em sítios favoráveis, ajudando na propagação da espécie.

Palavras-chaves: Floresta Atlântica, dispersão, leguminosa, cutia.
\end{abstract}

\begin{abstract}
This study aimed at evaluating the consumption, removal and secondary dispersal of seeds of Mucuna urens (Fabaceae - Faboideae) in an Atlantic Forest fragment in the Municipal Park of Lagoa do Peri, Florianópolis, SC. The biometric pattern and consumption by invertebrates of 100 seeds were evaluated. The removal and secondary dispersion were evaluated at 120 and 25 seeds, respectively. The average length, width and mass of seeds was $2.87 \pm 0.19 \mathrm{~cm}, 2.83 \pm 0.23$ $\mathrm{cm}$ and $5.04 \pm 1.60 \mathrm{~g}$, respectively, and forty-one percent of seeds were consumed by beetles Curculionidae, Scolytinae. There was no relationship between the length and the width of the seeds consumed and not consumed, and the consumed mass was significantly less than those not consumed, due to the fact that the Scolytinae feed the cotyledons. The agoutis (Dasyprocta azarae) removed $74.16 \%(\mathrm{n}=89)$ of the seeds. This rodent scattered $48 \%$ and buried $36 \%$ of the seeds, at an average distance of $8.06 \mathrm{~m}( \pm 7.46 \mathrm{~m})$ ranging from 1.00 to $35.90 \mathrm{~m}$. The secondary dispersion and hoarding seeds of M. urens by agoutis decreases the probability of predation by other vertebrates and invertebrates and increases the chances to occur the germination in favorable sites, helping in the propagation of the species. Key words: Atlantic Forest, dispersion, legumes, agoutis.
\end{abstract}

\section{Introdução}

A família Fabaceae é considerada uma das mais importantes dentre as angiospermas, com 630 gêneros e 18.000 espécies (Judd et al. 2009). Possui distribuição cosmopolita, estando amplamente presente nas regiões tropicais e, em menor número, nas regiões temperadas (Burkart 1979; Judd et al. 2009). Esta família é uma das responsáveis pela grande diversidade vegetal das florestas tropicais, tendo papel de destaque como elemento florístico nas principais formações vegetacionais brasileiras,

\footnotetext{
' Instituto de Pesquisas Jardim Botânico do Rio de Janeiro, Lab. Sementes, R. Pacheco Leão 915, Jardim Botânico, 22460-030, Rio de Janeiro, RJ.

${ }^{2}$ Universidade Federal de Santa Catarina, Depto. Ecologia e Zoologia, Centro de Ciências Biológicas, Campus Universitário, Trindade, 88040-900, Florianópolis, SC.

${ }^{3}$ Herbário Barbosa Rodrigues, Av. Cel. Marcos Konder, 88301-302, Itajaí, SC.

${ }^{4}$ Autora para correspondência: thalitagabriella@gmail.com
} 
principalmente em trechos da Mata Atlântica (Lima 2000). O gênero Mucuna Adans., em particular caracteriza-se por ser constituído por lianas e apresentar o fruto formado por duas valvas com cerdas urticantes (Agostini 2008). Algumas espécies desse gênero possuem valor econômico, sendo importantes para o ciclo do nitrogênio e como adubo orgânico (Oudhia 2001). De acordo com Forzza et al. (2010), seis espécies de Mucuna Adans. são encontradas no Brasil, entre elas Mucuna urens (L.) Medikus.

A distribuição de $M$. urens ocorre no Oceano Pacífico (Havaí), Caribe (Cuba, Haiti, Ilhas Trinidade e Tobago, Jamaica, Porto Rico, República Dominicana), América Central (Costa Rica, El Salvador, Guatemala, Nicarágua, Panamá) e América do Sul (Argentina, Brasil, Guiana, Guiana Francesa, Peru e Suriname) (Bisby et al. 2011). No Brasil, essa espécie ocorre na Floresta Amazônica e Mata Atlântica, e é nativa da Região Norte (Amazonas e Pará), Nordeste (Bahia e Maranhão), Centro-Oeste (Mato Grosso do Sul), Sudeste (Minas Gerais, Rio de Janeiro e São Paulo) e Sul (Paraná e Santa Catarina) (Forzza et al. 2010). É encontrada principalmente na restinga, mata de encosta, mata de galeria e em áreas de capoeira (Agostini 2008). Esta leguminosa produz muitos frutos e sementes (Agostini 2008) com grande valor nutricional (Adebooye \& Phillips 2006). O fruto pode produzir de uma a cinco sementes, e alcançar $30 \mathrm{~cm}$ de comprimento e $7 \mathrm{~cm}$ de largura quando ocorre o desenvolvimento de todas as sementes (Agostini 2008).

A dispersão das sementes é um processo demográfico chave na vida das plantas em florestas tropicais, determinando a variação na sobrevivência das sementes e, consequentemente, no estabelecimento de novas plantas adultas, sendo considerada uma das fases críticas no ciclo de vida das espécies vegetais (Brewer \& Webb 2001; Galetti et. al. 2006). As leguminosas, neste aspecto, apresentam grande diversidade de mecanismos de dispersão. Nas espécies que apresentam vagens achatadas, como no gênero Mucuna, as sementes são dispersas com a ação do vento quando os frutos são abertos (Judd et al. 2009).

Os roedores são um dos grupos de mamíferos frugívoros mais importantes nas florestas neotropicais e a maioria deles apresenta a dieta composta por frutos e sementes (Brewer \& Webb 2001; Andreazzi et al. 2009). Esses animais atuam na dispersão e na sobrevivência das sementes, influenciando a distribuição das espécies de plantas e a estrutura das comunidades vegetais (Forget 1992; Peres \& Baider 1997; Brewer \& Rejmánek 1999; Forget 2008). O comportamento de estocagem por roedores é um importante processo de dispersão secundária de sementes, pois diminui a probabilidade de predação e mantém as sementes viáveis, podendo ocorrer o recrutamento de novas plântulas (Brewer \& Rejmánek 1999; Brewer \& Webb 2001; Forget 2008). Roedores com comportamento dispersor estocador, como as cutias (Dasyprocta Illiger spp), frequentemente estão associados ao consumo de sementes nas florestas tropicais (Forget et al. 1992; Peres \& Baider 1997; Henry 1999). Elas consomem ou estocam as sementes para se alimentarem mais tarde, porém, muitas destas não são recuperadas e podem germinar, o que contribui na propagação das espécies vegetais (Peres \& Baider 1997; Fleury \& Galetti 2004).

Entre as causas de mortalidade de sementes, destacam-se o parasitismo, principalmente por fungos, e a predação, podendo ambas limitar o recrutamento de novos indivíduos em uma população. A predação de sementes pode ser dividida em duas etapas: pré-dispersão e pósdispersão. A predação pré-dispersão se refere ao ataque às sementes antes da dispersão, e é realizada principalmente por insetos, sendo um processo importante por diminuir a sobrevivência de plantas, podendo alcançar $80 \%$ de mortalidade das sementes produzidas (Janzen 1971, 1980). A família Fabaceae evidencia grandes perdas na produção de sementes nas florestas tropicais pelo ataque de predadores, principalmente por besouros (Green \& Palmbald 1975; Kaye 1999; Nogueira \& Arruda 2006; Ribeiro et al. 2007).

Nos trópicos, poucos estudos avaliaram efetivamente o destino das sementes removidas e mensuraram a predação e dispersão secundária em espécies com frutos secos, como as leguminosas. Desse modo, este trabalho teve como objetivo avaliar o consumo na copa, a remoção e dispersão de sementes de $M$. urens em um fragmento de Floresta Atlântica no Sul do Brasil. As questões deste estudo foram: (1) As sementes de $M$. urens sofrem consumo por invertebrados antes da dispersão? (2) Existe influência do tamanho e do peso das sementes no consumo por parte destes invertebrados? (3) Qual a participação dos vertebrados no processo de dispersão? (4) Qual o limite de dispersão das sementes da espécie? 


\section{Materiais e Métodos}

Área de estudo

O estudo foi realizado no Parque Municipal da Lagoa do Peri (PMLP), que está localizado no Sul da Ilha de Santa Catarina $\left(27^{\circ} 42\right.$ ' $30^{\prime \prime}$ e $27^{\circ} 46^{\prime} 30^{\prime \prime}$ s e $48^{\circ} 30^{\prime} 00^{\prime \prime}$ e $\left.48^{\circ} 33^{\prime} 30^{\prime \prime} \mathrm{W}\right)$. Com aproximadamente $20 \mathrm{~km}^{2}$, o parque abriga uma lagoa de água doce, com uma área de $5,07 \mathrm{~km}^{2}$, e vegetação constituída por fragmentos de restinga e Floresta Ombrófila Densa (CECCA 1997), que encontra-se em diferentes estágios de sucessão, com poucos locais com remanescentes primários de vegetação e que ocupa cerca de $14,3 \mathrm{~km}^{2}$ (Silva 2000). O estudo foi realizado em uma área de vegetação secundária de Floresta Ombrófila Densa de Encosta, em uma trilha com aproximadamente $2 \mathrm{~km}$ de extensão.

Sementes de $M$. urens foram coletadas dos frutos em 10 indivíduos da espécie no PMLP. Os padrões biométricos e o consumo por invertebrados foram avaliados em 100 sementes. $\mathrm{O}$ comprimento e a largura foram medidos com paquímetro, e o peso foi avaliado em balança eletrônica em laboratório. Posteriormente, as sementes foram abertas com o auxílio de martelo sobre uma prancha de madeira com $15 \times 15 \mathrm{~cm}$, para avaliação do consumo. Os invertebrados foram coletados no interior das sementes, conservados em álcool $70 \%$ e posteriormente identificados com a ajuda de especialistas da Universidade Federal de Santa Catarina. As sementes foram classificadas em: consumidas (apresentavam sinais de consumo dos cotilédones ou invertebrados no seu interior) e não consumidas (apresentavam os cotilédones intactos). As correlações entre o consumo e os padrões biométricos das sementes foram testadas pelo coeficiente de correlação de Spearman, pois a largura e comprimento não apresentaram normalidade segundo o teste D'Agostino (Zar 1996). Para avaliar se houve diferenças entre largura, comprimento e peso das sementes consumidas e não consumidas, foi realizado o teste de Mann-Whitney, utilizando o aplicativo BioEstat 5.0 (Ayres et al. 2007).

\section{Remoção de sementes}

Para avaliar a remoção de sementes de $M$. urens por vertebrados, foram utilizados 12 lotes com dez sementes $(n=120)$. Cada lote foi disposto próximo a um indivíduo de $M$. urens em frutificação, os quais estavam em uma trilha no PMLP. Os lotes foram marcados com uma pequena estaca vermelha para facilitar a posterior identificação. As avaliações foram realizadas a cada dez dias, durante um período de 40 dias, não havendo reposição de sementes. Em cada monitoramento foram verificadas a quantidade de sementes removidas e que permaneceram no local, sendo consideradas removidas aquelas que não eram encontradas a uma distância de, no mínimo, $0,5 \mathrm{~m}$ da posição inicial.

\section{Dispersão secundária de sementes}

Para avaliar a dispersão secundária por vertebrados, 25 sementes foram presas em carretéis de linha poliéster $(180 \mathrm{~m})$. Cada semente foi furada com furadeira elétrica portátil e amarrada à linha de um carretel. Cinco sementes foram amarradas ao tronco de cinco árvores que estavam próximas a indivíduos de $M$. urens em frutificação, ao longo da trilha selecionada no PMLP. A avaliação da dispersão foi realizada após dez dias da montagem do experimento. De acordo com o destino, as sementes foram classificadas em: (1) semente enterrada próxima à planta mãe (distância $\leq$ que 0,5 $\mathrm{m}$ ); (2) semente dispersa (distância $>$ que $0,5 \mathrm{~m}$ ) e enterrada; (3) semente retirada do carretel próximo à planta mãe; (4) semente retirada do carretel longe da planta mãe e (5) semente não removida. Foi medida a distância do deslocamento em linha reta, do local de origem até o local onde a semente foi enterrada (classe 2) ou até o local onde a linha foi cortada (classe 4). Os animais dispersores foram avaliados através de duas armadilhas fotográficas Tigrinus convencional modelo 4.0C. Elas foram fixadas próximas aos grupos de sementes dos experimentos de remoção e dispersão, com o foco direcionado para as sementes que foram colocadas no chão. As armadilhas permaneceram 40 dias (960 horas) em campo.

\section{Resultados}

$\mathrm{O}$ comprimento e a largura não diferiram entre as sementes consumidas e não consumidas $(\mathrm{Z}(\mathrm{U})=1,40, \mathrm{p}>0,05$ e $\mathrm{Z}(\mathrm{U})=0,56, \mathrm{p}>$ 0,05 , respectivamente), sendo que a massa das sementes não consumidas foi significativamente maior do que as que foram consumidas $(\mathrm{Z}(\mathrm{U})=$ $5,55, \mathrm{p}<0,0001$ ) (Tab. 1). Entre as 100 sementes avaliadas, $41 \%$ apresentavam sinais de consumo por invertebrados que, no caso, foram besouros Curculionidae, da subfamília Scolytinae. Também foi observado o uso das sementes na copa de $M$. urens como local de nidificação por formigas do gênero Crematogaster Lund. 
Tabela 1 - Padrões biométricos de sementes de Mucuna urens coletadas no Parque Municipal da Lagoa do Peri, Florianópolis, SC. Médias seguidas de letras diferentes na mesma coluna indicam diferenças estatisticamente significativas de acordo com o teste de Mann-Whitney $(\mathrm{n}=100, \mathrm{p}<0,05)$.

Table 1 - Biometric patterns of seeds of Mucuna urens collected in the Municipal Park of Lagoa do Peri, Florianópolis, SC. Means followed by different letters in the same column are significantly different, according to the Mann-Whitney test $(n=100, p<0,05)$.

\begin{tabular}{lccc}
\hline Sementes & Comprimento (cm) & Largura (cm) & Massa (g) \\
\hline Consumidas & $2,92 \pm 0,19$ a & $2,85 \pm 0,17$ a & $2,87 \pm 0,19$ \\
Não & $2,83 \pm 0,23$ a & $2,83 \pm 0,23$ a & $2,83 \pm 0,23$ \\
consumidas & & & \\
Média & $3,94 \pm 1,20 \mathrm{a}$ & $5,80 \pm 1,39$ b & $5,04 \pm 1,60$ \\
\hline
\end{tabular}

Na Figura 1a observa-se a percentagem acumulada de sementes removidas, sendo que a remoção ocorreu principalmente nos primeiros dez dias (Fig. 1b). A cutia (Dasyprocta azarae Lichtenstein) foi o único frugívoro registrado consumindo as sementes de $M$. urens no PMLP, sendo observada em $66 \%(\mathrm{n}=8)$ dos registros fotográficos. Entre as 25 sementes, 12 (48\%) foram dispersas pelas cutias (classes 2 e 4 ). Nove $(36 \%)$ sementes foram enterradas, sendo duas ( $8 \%)$ próximo (classe 1) e sete (28\%) longe da planta mãe (classe 2) (Fig. 2). A distância média das sementes dispersas e enterradas (classe 2) foi de $8,06 \mathrm{~m}( \pm 7,46 \mathrm{~m})$, variando de 1,00 a $35,90 \mathrm{~m}$. Não foi possível medir o deslocamento das sementes removidas e retiradas do carretel (classe 4), que representaram $20 \%(n=5)$, contudo, mediu-se até onde o fio foi rompido, tendo-se uma distância média de $11 \mathrm{~m}( \pm 8,46 \mathrm{~m})$, com uma variação entre 1,9 e 21,8 m. Entre as sementes dispersas pela cutia, não foram observadas marcas de consumo.

\section{Discussão}

Como não houve interferência do comprimento e da largura no consumo por parte das sementes de $M$. urens, acredita-se que esses dois parâmetros não devem influenciar na escolha da oviposição dos escolitíneos, o que difere do proposto por Janzen (1969), que sugere que o tamanho do fruto ou da semente são fatores que afetam a escolha do sítio de oviposição por insetos. Como em $M$. urens, também não houve seleção das sementes maiores para a oviposição dos insetos brocadores em Sophora tomentosa L. (Fabaceae) (Paise 2007).

A massa das sementes de $M$. urens interferiu no ataque dos escolitíneos, e pelo cotilédone ser o principal constituinte da semente dessa espécie, correspondendo de 55,3 a 71,9\% da sua massa total (Adebooye \& Phillips 2006), sugere-se que a menor massa das sementes infestadas pelos escolitíneos seja devido ao consumo dos cotilédones por estes besouros. Nogueira \& Arruda (2006) observaram que as sementes predadas de $S$. tomentosa tinham um peso $30 \%$ menor em relação às sementes não predadas, valor semelhante ao encontrado no presente estudo para $M$. urens, em que o peso médio das sementes predadas foi $32 \%$ menor em relação as não predadas.
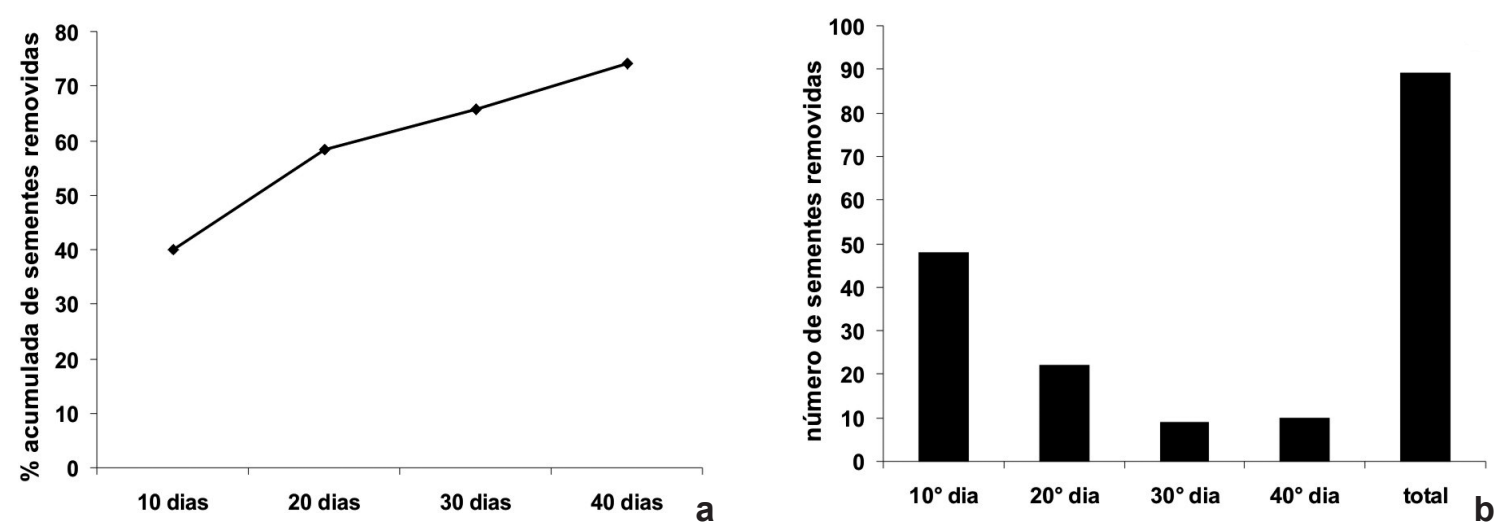

Figura 1 - Porcentagem acumulada (a) e número de sementes removidas (b) de Mucuna urens (L.) Medikus por cutias (Dasyprocta azarae Lichtenstein), no Parque Municipal da Lagoa do Peri, Florianópolis (SC).

Figure 1 - Cumulative percentage (a) and number of seeds removed (b) of Mucuna urens (L.) Medikus by agoutis (Dasyprocta azarae Lichtenstein), in the Municipal Park of Lagoa do Peri, Florianópolis (SC). 


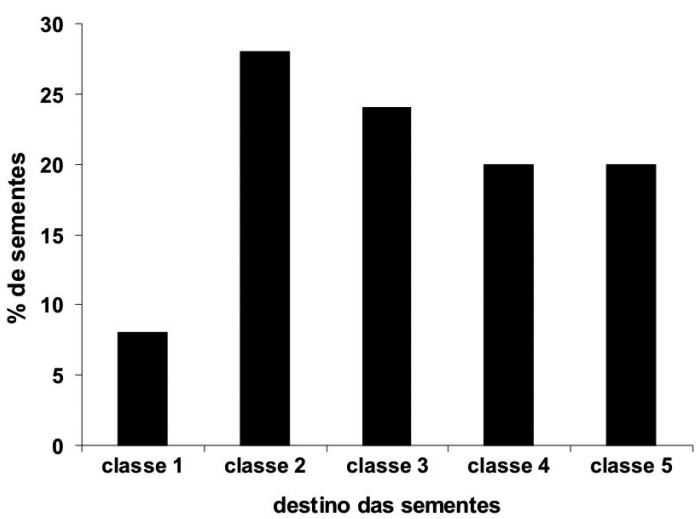

Figura 2 - Porcentagem de sementes de Mucuna urens (L.) Medikus dispersas por cutias (Dasyprocta azarae Lichtenstein), no Parque Municipal da Lagoa do Peri, Florianópolis (SC). Legenda: classe 1- sementes enterradas próximas à planta mãe (distância $\leq$ que $0,5 \mathrm{~m}$ ); classe 2 - sementes dispersas (distância > que 0,5 m) e enterradas; classe 3 - sementes retiradas do carretel próximo à planta mãe; classe 4 - sementes retiradas do carretel longe da planta mãe; classe 5 - sementes não removidas.

Figure 2-Percentage of Mucuna urens (L.) Medikus seeds dispersed by agoutis (Dasyprocta azarae Lichtenstein), in the Municipal Park of Lagoa do Peri, Florianópolis (SC). Legend: class 1 - seeds buried near the mother plant (distance $\leq 0.5 \mathrm{~m}$ ), class 2 - dispersed seeds (distance $>0.5$ $\mathrm{m}$ ) and buried; class 3 - seeds removed from the reel near the mother plant; class 4 - seeds removed from the reel away from the mother plant, class 5 - seeds not removed.

Janzen (1969) sugere que, para as leguminosas, quanto menor a semente e quanto maior o número e peso das sementes por metro cúbico de copa, maior será a percentagem de predação por bruquíneos (Coleoptera, Chrysomelidae, Bruchinae). Em relação às espécies com sementes grandes (massa $>5$ $\mathrm{g}$ ), estas produziriam menos propágulos, que teriam uma maior concentração de defesas químicas contra os predadores. Contudo, como no presente estudo, Janzen (1969) também observou a predação nas sementes de $M$. urens por escolitíneos, acreditando que isso tenha ocorrido devido a essa leguminosa apresentar uma baixa ou ineficiente produção de metabólitos secundários de defesa contra esses besouros. Já Young (1983) não encontrou sinais de predação por insetos nas sementes dessa leguminosa, e acredita que a causa provável da mortalidade tenha sido o ataque por esquilo. No PMLP, onde não há registro desses roedores (Graipel et al. 2001), não foram observados sinais de predação das sementes dispersas pela cutia.
Em M. urens, o consumo de sementes por escolitíneos no período de pré-dispersão (41\%) pode ser um fator limitante à disponibilidade de sementes viáveis para o recrutamento de novos indivíduos na população. Scolytinae está entre as principais subfamílias de Curculionidae consumidoras de estruturas vegetais, e tanto as larvas como os adultos podem viver no interior das sementes (Costa Lima 1952; Berti Filho 1979), e no caso de $M$. urens, somente as larvas foram encontradas nas sementes.

Como em M. urens, em outras leguminosas também foram encontradas altas taxas de predação por besouros. O bruquíneo Acanthoscelides fraterculus Horn foi responsável pela predação de 74\% e 60\% das sementes de Astragalus cibarius E. Sheld. e Astragalus utahensis (Torr.) Torr. \& A. Gray, respectivamente (Green \& Palmbald 1975), e 60,3\% das sementes de Astragalus australis var. olympicus Isely (Kaye 1999).

A ocupação das sementes por formigas do gênero Crematogaster demonstra o uso secundário desse recurso pela fauna, utilizando-as como local de nidificação. Acredita-se que as formigas ocupam o interior das sementes que apresentam um oríficio ou abertura gerada pela saída dos besouros escolitineos ou pelo ataque de fungos e/ou microrganismos. Assim, essas formigas não seriam consideradas consumidoras de sementes de M. urens. Conforme Silva et al. (2009), muitas espécies de formigas constroem seus próprios ninhos, enquanto outras utilizam cavidades naturais já existentes. Esses autores encontraram 11 espécies e morfoespécies em seis gêneros de Formicidae utilizando a cavidade interna de sementes de Syagrus romanzoffiana (Cham.) Glasm. (Arecaceae) para nidificação.

O registro do consumo de sementes de $M$. urens por cutias destaca esta leguminosa como importante fonte de alimento para a fauna, uma vez que suas sementes têm grande valor nutricional (Adebooye \& Phillips 2006), destacando também o papel dos dispersores estocadores ("scatterhoarding behavior") na dispersão desta espécie. O consumo de sementes de leguminosas por cutias já havia sido demonstrado por outros trabalhos (Henry 1999; Silvius \& Fragoso 2003; Guimarães Jr. et al. 2006; McWilliams 2009). Estudos também mostraram a alta percentagem de sementes grandes deslocadas por esses roedores, sendo que em Gustavia superba (Kunth) O. Berg (Lecythidaceae), 
$85,5 \%$ das sementes foram removidas após 28 dias (Forget et al. 1992), e 87,6\% das sementes da castanheira-do-Pará (Bertholletia excelsa Bonpl., Lecythidaceae) após duas semanas, sendo a maioria delas deslocadas em apenas quatro dias (Peres \& Baider 1997).

A distância em que as sementes de $M$. urens foram dispersas e enterradas pela cutia foram superiores ao encontrado para as sementes da castanheira-do-Pará (B. excelsa), que foram enterradas por esse roedor a uma distância média de cinco metros do ponto amostral, sendo que raramente ultrapassou $20 \mathrm{~m}$ (Peres \& Baider 1997). Todas as sementes de M. urens enterradas (36\%) estavam intactas, o que aumenta consideravelmente as chances de germinação. Peres \& Baider (1997) observaram que $65 \%$ das castanhas-do-Pará estocadas pela cutia não apresentavam sinais de consumo e que, para cada semente consumida por esse roedor, de três a sete foram enterradas. Assim, observa-se que a cutia desempenha um importante papel como dispersor secundário, principalmente por estar presente em grande abundância tanto em florestas primárias como em vegetações perturbadas (Henry 1999).

Até o presente estudo, havia sido relatado que $M$. urens apresentava dispersão primária por autocoria e secundária por hidrocoria (Reis, A. comunicação pessoal). A descoberta de que as sementes dessa leguminosa também são dispersas secundariamente por animais, mostra que essa planta apresenta duas síndromes de dispersão secundária, hidrocoria e zoocoria, o que aumenta as chances de propagação da espécie, tanto pela água como pelo solo. A dispersão e a estocagem das sementes de $M$. urens por cutias diminui a probabilidade de ocorrer a predação por outros vertebrados e invertebrados e aumenta as chances de germinação e recrutamento de novas plântulas.

\section{Agradecimentos}

Ao Programa de Pós-Graduação em Biologia Vegetal (PPGBVE/UFSC) e ao biólogo André Ganzarolli Martins, que identificou os coleópteros.

\section{Referências}

Ayres, M.; Ayres Jr., M.; Ayres, D.L.; Santos, A.S. 2007. Biostat: aplicações estatísticas nas areas das ciências médicas. Mamirauá, Belém, 364p.

Adebooye, O.C. \& Phillips, O.T. 2006. Studies on seed characteristics and chemical composition of three morphotypes of Mucuna urens (L.) Medikus Fabaceae. Food Chemistry 95: 658-663.

Agostini, K. 2008. Ecologia da reprodução de duas espécies de Mucuna (Fabaceae, Faboideae, Phaseoleae) embriologia, citogenética e genética populacional - do litoral norte de São Paulo. Tese de Doutorado. Universidade Estadual de Campinas, São Paulo. 178p.

Andreazzi, C.S.; Pires, A.S. \& Fernandez, F.A.S. 2009. Mamíferos e palmeiras neotropicais: interações em paisagens fragmentadas. Oecologia Brasiliensis 13: 554-574.

Berti Filho, E. 1979. Coleópteros de importância florestal: 1 - Scolytidae. IPEF 19: 39-43.

Bisby, F.; Roskov, Y.; Schrire, B.; White, R. \& Zarrucchi, J. 2011. The Legume Web from the ILDIS World Database of Legumes, version 10.01 of the ILDIS World Database of Legumes. Disponível em: $<$ http://www.ildis.org/LegumeWeb $>$. Acesso em 10 Nov 2011.

Brewer, S.W. \& Rejmánek, M. 1999. Small rodents as significant dispersers of tree seeds in a Neotropical forest. Journal of Vegetation Science 10: 165-174.

Brewer, S.W. \& Webb, M.A.H. 2001. Ignorant seed predators and factors affecting the seed survival of a tropical palm. Oikos 93: 32-41.

Burkart, A. 1979. Leguminosas - Mimosoideas. In: Reitz, R. (ed.). Flora Ilustrada Catarinense. Itajaí, SC. 304p.

CECCA - Centro de Estudos Cultura e Cidadania. 1997. Unidades de conservação e áreas protegidas da Ilha de Santa Catarina: caracterização e legislação. Ed. Insular. 160p.

Costa Lima, A. 1952. Insetos do Brasil. Coleópteros. Escola Nacional de Agronomia, Série didática 9, Rio de Janeiro. 372p.

Fleury, M. \& Galetti, M. 2004. Effects of microhabitat on palm seed predation in two forest fragments in southeast Brazil. Acta Oecologica 26: 179-184.

Forget, P.M. 1992. Seed removal and seed fate in Gustavia superba (Lecythidaceae). Biotropica 24: 408-414.

Forget, P.M. 2008. Survival and scatterhoarding of frugivores-dispersed seeds as a function of forest disturbance. Biotropica 40: 380-385.

Forzza et al. 2010. Catálogo de plantas e fungos do Brasil. Vol. 2. Instituto de Pesquisas Jardim Botânico do Rio de Janeiro, Rio de Janeiro. 830p.

Galetti, M.; Donatti, C.I.; Pires, A.S.; Guimarães JR., P.R. \& Jordano, P. 2006. Seed survival and dispersal of an endemic Atlantic forest palm: the combined effects of defaunation and forest fragmentation. Botanical Journal of the Linnean Society 151: 141-149.

Graipel, M.E.; Cherem, J.J. \& Ximenez, A. 2001. Mamíferos terrestres não voadores da Ilha de Santa Catarina, no sul do Brasil. Biotemas 14: 109-140.

Green, T.W. \& Palmbald, I.G. 1975. Effects of insect seed predators on Astragalus cibarius and Astragalus utahensis (Leguminosae). Ecology 56: 1435-1440. 
Guimarães Jr., P.R.; Kubota, U.; Gomes, B.Z.; Fonseca, R.L.; Bottcher, C. \& Galetti, M. 2006. Testing the quick meal hypothesis: the effect of pulp on hoarding and seed predation of Hymenaea courbaril by redrumped agoutis (Dasyprocta leporina). Austral Ecology 31: 95-98.

Henry, O. 1999. Frugivory and the importance of seeds in the diet of the orange-rumped agouti (Dasyprocta leporina) in French Guiana. Journal of Tropical Ecology 15: 291-300.

Janzen, D.H. 1969. Seed-eaters versus seed-size, number, toxicity and dispersal. Evolution 23: 1-27.

Janzen, D.H. 1971. Escape of Cassia grandis L. beans from predators in time and space. Ecology 52: 964-979.

Janzen, D.H. 1980. Specificity of seed-attacking bettles in a Costa Rican deciduous forest. Journal of Ecology 68: 929-952.

Judd, W.S.; Campbell, C.S.; Kellogg, E.A.; Stevens, P.F. \& Donoghue, M.J. 2009. Sistemática vegetal. Um enfoque filogenético. $3^{\mathrm{a}}$ ed. Artmed, Porto Alegre. 632p.

Kaye, T.N. 1999. From flowering to dispersal: reproductive ecology of an endemic plant, Austragalus australis var. olympicus (Fabaceae). American Journal of Botany 86: 1248-1256.

Lima, H.C. 2000. Leguminosas da Mata Atlântica: uma análise da riqueza, padrões de distribuição geográfica e similaridades florísticas em remanescentes florestais do estado do Rio de Janeiro. Tese de Doutorado. Universidade Federal do Rio de Janeiro. 141p.

McWilliams, D.A. 2009. Determinants for the diet of captive agoutis (Dasyprocta spp.). Veterinary Clinics of North America 12: 279-286.

Nogueira, E.M.L. \& Arruda, V.L.V. 2006. Frutificação e danos em frutos e sementes de Sophora tomentosa L. (Leguminosae, Papilionoideae) em restinga da praia da Joaquina, Florianópolis, SC. Biotemas 19: 41-48.

Oudhia, P. 2001. Kapikachu or Cowhage (Mucuna pruriens). Disponível em $<\mathrm{http}$ ://www.celestine-india. com/pankajoudhia>. Acesso em 10 Out 2011.

Paise, G. 2007. Aborto seletivo e predação de sementes no feijão-da-praia Sophora tomentosa (Fabaceae). In: Machado, G.; Prado, P.I.K. \& Oliveira, A.A. (eds.). Livro do curso de campo "Ecologia da Mata Atlântica”. USP, São Paulo. Pp. 1-5.

Peres, C.A. \& Baider, C. 1997. Seed dispersal, spatial distribution and population structure of Brazilnut trees (Bertholletia excelsa) in southeasterm Amazonia. Journal of Tropical Ecology 13: 595-616.

Ribeiro, M.L.; Sales, V.A.; Miranda, F.S. \& Aguiar, C.E. 2007. Influência da predação de sementes na germinação de leguminosas (Fabaceae) no Cerrado. Revista Brasileira de Biociências 5: 279-281.

Silva, A.A.S. 2000. Parque Municipal da Lagoa do Peri: subsídios para o gerenciamento ambiental. Dissertação de Mestrado. Universidade Federal de Santa Catarina, Florianópolis. 130p.

Silva, F.R.; Begnini, R.M.; Klier, V.A.; Scherer, K.Z.; Lopes, B.C. \& Castellani, T.T. 2009. Utilização de sementes de Syagrus romanzoffiana (Arecaceae) por formigas em floresta secundária no sul do Brasil. Neotropical Entomology 38: 873-875.

Silvius, K.M. \& Fragoso, J.M.V. 2003. Red-rumped agouti (Dasyprocta leporina): home range use in an Amazonian Forest: implications for the aggregated distribution of forest trees. Biotropica 35: 74-83.

Young, A.M. 1983. Seed mortality and recruitment in the forest canopy vine Mucuna urens (Leguminosae) in the central highlands of Costa Rica. Brenesia 21: 13-25.

Zar, J.H. 1996. Biostatistical analysis. $3^{\text {rd }}$ ed. Prentice Hall, New Jersey. 620p. 
